\title{
Transcriptome Analysis Combined With Glutathione S-transferase 1 Gene Family Whole-genome Identification Reveals the Mechanism Underlying the Exogenous Application of 5-aminolevulinic Acid to Alleviate Low Temperature Stress in Solanum Lycopersicum Seedling
}

\section{Zhengda Zhang}

Northwest A\&F University: Northwest Agriculture and Forestry University

Tao Liu

Shenyang Agricultural University

\section{Zhen Kang}

Northwest A\&F University: Northwest Agriculture and Forestry University

Jiwen Xu

Northwest A\&F University: Northwest Agriculture and Forestry University

Shichun Yang

Northwest A\&F University: Northwest Agriculture and Forestry University

Yuhui Zhang

Northwest A\&F University: Northwest Agriculture and Forestry University

Yongshuai Wen

Northwest A\&F University: Northwest Agriculture and Forestry University

Zijian Xu

Northwest A\&F University: Northwest Agriculture and Forestry University

Jianyu Yang

Northwest A\&F University: Northwest Agriculture and Forestry University

Tianlai Li

Shenyang Agricultural University

Xiaohui Hu ( $\square$ hxh1977@163.com )

Northwest A\&F University: Northwest Agriculture and Forestry University https://orcid.org/0000-0002$1236-9820$

Research article 
Keywords: low temperature, glutathione S-transferase, RNA sequencing, Solanum lycopersicum, 5aminolevulinic acid, reactive oxygen species

Posted Date: September 10th, 2020

DOI: https://doi.org/10.21203/rs.3.rs-67624/v1

License: (c) (1) This work is licensed under a Creative Commons Attribution 4.0 International License. Read Full License 


\section{Abstract}

Background: Transcriptome sequencing was conducted to screen out genes that actively respond to exogenous 5-aminolevulinic acid (ALA) induction under low temperature stress. The study used two versions of the tomato genome database to strictly screen and identify tomato glutathione S-transferase (GST) gene families and carried out the related bioinformatics analysis of tomato GST gene family. The expression pattern of SIGST genes induced by exogenous application ALA under low temperature stress was also analysed. Related physiological indicators were determined, and related chemical stains were performed.

Results: RNA sequencing (RNA-seq) results showed that the expression of SIGST gene was different under various treatments, and a large number of SIGST genes widely responded to ALA induction under low temperature stress. Sixty-nine full-length GST genes were identified by screening the two versions of tomato genome databases combined with protein domain analysis. Analysis of gene family phylogenetic tree divided the tomato GST gene family into eight subfamilies. Tandem replication of genes is one of the driving forces for the evolution of tomato GST gene family, and a large number of cis-acting elements are related to stress resistance on the promoter of the GST gene family. Exogenous ALA application under low temperature stress induces a broad response of tomato leaf SIGST gene (qRT-PCR verification), increases GST activity and decreases reactive oxygen species (ROS) accumulation.

Conclusions: RNA sequencing results revealed that a large number of tomato GST genes are differentially expressed, and Sixty-nine GSTs are identified in the tomato genome. Tandem replication of genes is the driving force for 68 the evolution of tomato GST family, and the promoter contains a large number of cisacting elements related to stress resistance. Test results show that exogenous ALA induces the expression of SIGST genes under low temperature stress, thereby increasing GST activity to eliminate the ROS produced under low temperature stress and increase the tomato tolerance.

\section{Full Text}

This preprint is available for download as a PDF.

\section{Tables}

Table 1 Summary of RNA-seq data sets 


\begin{tabular}{cccccccc}
\hline Samples & Clean reads & Clean bases & GC $(\%)$ & Q30 (\%) & Total reads & Mappable reads & Mapping rate (\%) \\
\hline CK1 & 8364597146 & 27936903 & 43.31 & 94.58 & 55873806 & 49430367 & 88.47 \\
CK2 & 8317330184 & 27789453 & 43.47 & 94.69 & 55578906 & 48787623 & 87.78 \\
CK3 & 7691554432 & 25699897 & 43.48 & 94.93 & 51399794 & 45225767 & 87.99 \\
ALA1 & 8026187750 & 26810222 & 43.32 & 94.56 & 53620444 & 47022467 & 87.70 \\
ALA2 & 7404555476 & 24737565 & 43.25 & 94.53 & 49475130 & 43805327 & 88.54 \\
ALA3 & 7617858308 & 25449304 & 43.59 & 94.33 & 50898608 & 43834104 & 86.12 \\
LT1 & 6900841340 & 23049776 & 42.94 & 94.41 & 46099552 & 40410994 & 87.66 \\
LT2 & 7270022498 & 24279980 & 42.92 & 94.24 & 48559960 & 42743978 & 88.02 \\
LT3 & 11240523464 & 37570881 & 44.53 & 94.26 & 75141762 & 58948227 & 78.45 \\
LTA1 & 6799325530 & 22716030 & 43.22 & 94.29 & 45432060 & 39090523 & 86.04 \\
LTA2 & 7234826466 & 24174036 & 43.25 & 94.46 & 48348072 & 41217602 & 85.25 \\
LTA3 & 6918924098 & 23158144 & 43.41 & 94.48 & 46316288 & 39858708 & 86.06 \\
\hline
\end{tabular}

Table 2 Identified GST genes in tomato with information and location predictions 


\begin{tabular}{|c|c|c|c|c|c|c|c|c|c|c|c|}
\hline \multirow[t]{2}{*}{ No. } & \multirow[t]{2}{*}{ Gene Name } & \multirow{2}{*}{$\begin{array}{c}\text { Gene ID } \\
\text { (ITAG 2.40) }\end{array}$} & \multirow{2}{*}{$\begin{array}{l}\text { Gene ID } \\
\text { (ITAG 4.0) }\end{array}$} & \multicolumn{3}{|c|}{ Length (bp) } & \multirow{2}{*}{$\begin{array}{c}\text { MW } \\
(\mathrm{kDa})\end{array}$} & \multirow[t]{2}{*}{ Exons } & \multirow[t]{2}{*}{$\mathrm{pI}$} & \multirow[t]{2}{*}{ Protein(aa) } & \multirow[t]{2}{*}{ Localization } \\
\hline & & & & Gene & cDNA & CDS & & & & & \\
\hline 1 & SIGSTF1 & Solyc09g074850.2 & Solyc09g074850.4.1 & 2416 & 1147 & 814 & 22.94 & 6 & 5.42 & 200 & $\mathrm{Cy}^{\mathrm{a}, \mathrm{b}}$ \\
\hline 2 & SIGSTF2 & Solyc $02 g 081340.2$ & Solyc02g081340.3.1 & 1359 & 959 & 693 & 26.55 & 3 & 5.99 & 230 & $\mathrm{Cy}^{\mathrm{a}, \mathrm{b}}$ \\
\hline 3 & SIGSTF3 & Solyc06g009020.2 & Solyc06g009020.2.1 & 2217 & 1403 & 642 & 23.72 & 3 & 5.98 & 213 & $\mathrm{Cy}^{\mathrm{a}}, \mathrm{Cp} \mathrm{p}^{\mathrm{b}}$ \\
\hline 4 & SIGSTF4 & Solyc12g094430.1 & Solyc12g094430.1.1 & 2156 & 666 & 666 & 25.07 & 3 & 6.85 & 221 & $\mathrm{Cy}{ }^{\mathrm{a}}, \mathrm{Nu}^{\mathrm{a}}, \mathrm{Cp} \mathrm{p}^{\mathrm{b}}$ \\
\hline 5 & SIGSTF5 & Solyc06g009040.2 & Solyc06g009040.4.1 & 1953 & 849 & 639 & 23.86 & 3 & 6.08 & 212 & $\mathrm{Cy}{ }^{\mathrm{a}}, \mathrm{Cp}{ }^{\mathrm{b}}$ \\
\hline 6 & SIGSTT1 & Solyc08g080900.2 & Solyc08g080900.3.1 & 4012 & 1198 & 753 & 28.58 & 7 & 9.25 & 250 & $\mathrm{Cy}{ }^{\mathrm{a}, \mathrm{b}}$ \\
\hline 7 & SIGSTT2 & Solyc 08g080910.2 & Solyc08g080910.3.1 & 3707 & 1369 & 753 & 28.69 & 7 & 9.21 & 250 & $\mathrm{Cy}{ }^{a, b}, N^{b}$ \\
\hline 8 & SIGSTT3 & Solyc12g056250.1 & Solyc12g056250.2.1 & 2138 & 711 & 711 & 26.55 & 7 & 6.06 & 236 & $\mathrm{Cy}^{\mathrm{a}}, \mathrm{Mt}^{\mathrm{b}}$ \\
\hline 9 & SIMGST1 & Solyc $04 g 081740.2$ & Solyc $04 g 081740.3 .1$ & 4097 & 1497 & 1158 & 43.48 & 6 & 9.14 & 386 & $\mathrm{Mt}^{\mathrm{a}}, \mathrm{Cp}{ }^{\mathrm{a}}, \mathrm{Cy}{ }^{\mathrm{b}}$ \\
\hline 10 & $S I E F 1 B \gamma 1$ & Solyc11g028100.1 & Solyc11g028100.2.1 & 2091 & 1245 & 1245 & 47.33 & 6 & 5.94 & 414 & $\mathrm{Cp}^{\mathrm{a}}, \mathrm{Cy}{ }^{\mathrm{b}}$ \\
\hline 11 & $S I E F 1 B \gamma 2$ & Solyc06g011280.2 & Solyc06g011280.3.1 & 3077 & 1675 & 1242 & 47.05 & 8 & 5.66 & 413 & $\mathrm{Cp} \mathrm{p}^{\mathrm{ab}}$ \\
\hline 12 & SIGSTL1 & Solyc04g009530.2 & Solyc04g009530.4.1 & 5207 & 1191 & 888 & 33.52 & 10 & 6.25 & 295 & $\mathrm{Cy}^{\mathrm{a}}, \mathrm{Cp} \mathrm{p}^{\mathrm{b}}$ \\
\hline 13 & SIGSTL2 & Solyc09g007150.2 & Solyc09g007150.3.1 & 3633 & 953 & 717 & 27.59 & 10 & 4.97 & 238 & $\mathrm{Cy}{ }^{\mathrm{a}, \mathrm{b}}$ \\
\hline 14 & SIGSTL3 & Solyc10g084400.1 & Solyc10g084400.2.1 & 2340 & 708 & 708 & 27.18 & 10 & 5.06 & 235 & $\mathrm{Cyt}^{\mathrm{a}, \mathrm{b}}$ \\
\hline 15 & SIGSTL4 & Solyc12g044520.1 & Solyc12g044520.2.1 & 3780 & 720 & 720 & 27.84 & 10 & 5.52 & 239 & $\mathrm{Cy}^{\mathrm{a}}, \mathrm{Nu}{ }^{\mathrm{b}}$ \\
\hline 16 & SIGSTL5 & Solyc12g044530.1 & Solyc12g044530.3.1 & 3744 & 789 & 789 & 30.36 & 9 & 6.84 & 262 & $\mathrm{Cy}{ }^{\mathrm{a}}, \mathrm{Mt}^{\mathrm{a}}, \mathrm{Cp}{ }^{\mathrm{b}}$ \\
\hline 17 & SIGSTL6 & Solyc00g007030.1 & Solyc02g038818.1.1 & 3746 & 720 & 720 & 27.98 & 10 & 5.73 & 239 & $\mathrm{Cy}^{\mathrm{a}}, \mathrm{Nu}{ }^{\mathrm{b}}$ \\
\hline 18 & SIGSTL 7 & Solyc $00 \mathrm{~g} 007040.1$ & None & 2975 & 708 & 708 & 27.76 & 8 & 8.86 & 235 & $\mathrm{Cy}{ }^{\mathrm{a}, \mathrm{b}}, \mathrm{Mt}^{\mathrm{a}}$ \\
\hline 19 & SIDHAR1 & Solyc $05 g 054760.2$ & Solyc $05 \mathrm{~g} 054760.4 .1$ & 4424 & 1162 & 633 & 23.55 & 6 & 6.32 & 210 & $\mathrm{Cy}{ }^{\mathrm{a}, \mathrm{b}}$ \\
\hline 20 & SIDHAR2 & Solyc11g011250.1 & Solyc11g011250.3.1 & 5344 & 807 & 807 & 29.85 & 6 & 8.59 & 268 & $\mathrm{Mt}^{\mathrm{a}}, \mathrm{Cp}^{\mathrm{ab}}$ \\
\hline 21 & SIGSTZ1 & Solyc01g091330.2 & Solyc01g091330.4.1 & 6206 & 1195 & 855 & 32.14 & 10 & 6.97 & 284 & $\mathrm{Cy}{ }^{\mathrm{a}}, \mathrm{Cp} \mathrm{p}^{\mathrm{b}}$ \\
\hline 22 & SIGSTZ2 & Solyc01g102660.2 & Solyc01g102660.4.1 & 5178 & 1262 & 669 & 25.27 & 11 & 5.45 & 222 & $\mathrm{Cy}{ }^{\mathrm{a}, \mathrm{b}}$ \\
\hline 23 & SIGSTU1 & Solyc12g036560.1 & Solyc12g036560.2.1 & 333 & 333 & 333 & 12.89 & 1 & 6.72 & 110 & $\mathrm{Mt}^{\mathrm{a}}, \mathrm{Nu}{ }^{\mathrm{b}}$ \\
\hline 24 & SIGSTU2 & Solyc09g063150.2 & Solyc09g063150.3.1 & 1775 & 889 & 663 & 25.89 & 2 & 6.19 & 220 & $\mathrm{Cy}{ }^{\mathrm{a}, \mathrm{b}}, \mathrm{Cp}^{\mathrm{a}}$ \\
\hline 25 & SIGSTU3 & Solyc09g011490.2 & Solyc09g011490.3.1 & 1023 & 882 & 660 & 24.96 & 2 & 5.75 & 219 & $\mathrm{Cy}$ a,b \\
\hline 26 & SIGSTU4 & Solyc09g011500.2 & Solyc09g011500.3.1 & 1584 & 922 & 669 & 25.42 & 2 & 5.27 & 222 & $\mathrm{Cy}{ }^{\mathrm{a}}, \mathrm{Cp}{ }^{\mathrm{b}}$ \\
\hline 27 & SIGSTU5 & Solyc09g011520.2 & Solyc09g011520.3.1 & 1118 & 1021 & 678 & 26.03 & 2 & 6.02 & 225 & $\mathrm{Cy}^{\mathrm{a}}, \mathrm{Cp}^{\mathrm{a}}$ \\
\hline 28 & SIGSTU6 & Solyc09g011540.2 & Solyc09g011540.2.1 & 1031 & 907 & 669 & 25.45 & 2 & 5.05 & 222 & $\mathrm{Cy}^{\mathrm{a}}, \mathrm{Nu}{ }^{\mathrm{b}}$ \\
\hline 29 & SIGSTU7 & Solyc09g011550.2 & Solyc09g011550.3.1 & 1075 & 864 & 660 & 25.36 & 2 & 5.45 & 219 & $\mathrm{Cy} \mathrm{a}^{\mathrm{a} b}$ \\
\hline 30 & SIGSTU8 & Solyc09g011560.2 & Solyc09g011560.3.1 & 1499 & 1043 & 654 & 25.16 & 2 & 5.70 & 217 & $\mathrm{Cy}^{\mathrm{a}, \mathrm{b}}$ \\
\hline 31 & SIGSTU9 & Solyc09g011570.2 & None & 1334 & 909 & 633 & 24.50 & 2 & 5.87 & 210 & $\mathrm{Cy}^{\mathrm{a}}, \mathrm{Cp} \mathrm{p}^{\mathrm{b}}$ \\
\hline 32 & SIGSTU10 & Solyc09g011580.2 & None & 1290 & 9115 & 654 & 25.37 & 2 & 5.17 & 217 & $\mathrm{Cy} \mathrm{a}^{\mathrm{a}} \mathrm{b}$ \\
\hline 33 & SlGSTU11 & Solyc09g011590.2 & Solyc09g011590.4.1 & 1555 & 903 & 654 & 25.11 & 2 & 5.30 & 217 & $\mathrm{Cy}{ }^{\mathrm{a}, \mathrm{b}}$ \\
\hline 34 & SIGSTU12 & Solyc $09 g 011600.2$ & Solyc09g011600.3.1 & 1617 & 1033 & 654 & 25.27 & 2 & 5.90 & 217 & $\mathrm{Cy}^{\mathrm{a}}, \mathrm{Nu}{ }^{\mathrm{b}}$ \\
\hline 35 & SIGSTU13 & Solyc09g011620.1 & Solyc09g011620.2.1 & 811 & 648 & 648 & 24.76 & 2 & 5.39 & 215 & $\mathrm{Cy}{ }^{\mathrm{a}}, \mathrm{Cp}{ }^{\mathrm{b}}$ \\
\hline 36 & SIGSTU14 & Solyc09g011630.2 & Solyc09g011630.3.1 & 1278 & 917 & 657 & 24.86 & 2 & 5.53 & 218 & $\mathrm{Cy} \mathrm{a}^{\mathrm{a}, \mathrm{b}}$ \\
\hline 37 & SlGSTU15 & Solyc09g011640.2 & Solyc09g011640.5.1 & 1493 & 891 & 663 & 25.27 & 2 & 5.82 & 220 & $\mathrm{Cy}^{\mathrm{a}}, \mathrm{Mt}^{\mathrm{b}}$ \\
\hline 38 & SIGSTU16 & Solyc01g081260.1 & Solyc01g081260.2.1 & 884 & 381 & 381 & 15.09 & 2 & 7.87 & 126 & $\mathrm{Cy}^{\mathrm{b}}, \mathrm{Mt}^{\mathrm{a}}$ \\
\hline 39 & SIGSTU17 & Solyc01g081270.2 & Solyc01g081270.2.1 & 1342 & 1181 & 672 & 25.94 & 2 & 5.47 & 223 & $\mathrm{Cy}$ \\
\hline 40 & SIGSTU18 & Solyc09g091130.2 & Solyc09g091130.4.1 & 1006 & 684 & 675 & 26.40 & 2 & 7.70 & 224 & $\mathrm{Cy}{ }^{\mathrm{a}, \mathrm{b}}, \mathrm{Cp}^{\mathrm{a}}$ \\
\hline 41 & SIGSTU19 & Solyc12g011300.1 & Solyc12g011300.2.1 & 1128 & 663 & 663 & 25.64 & 2 & 5.39 & 220 & $\mathrm{Cy}$ \\
\hline 42 & SIGSTU20 & Solyc12g011310.1 & Solyc12g011310.2.1 & 1213 & 660 & 660 & 25.62 & 2 & 6.91 & 219 & $\mathrm{Cy}^{\mathrm{a}}, \mathrm{Nu}{ }^{\mathrm{b}}$ \\
\hline 43 & SlGSTU21 & Solyc12g011320.1 & Solyc12g011320.2.1 & 1187 & 660 & 660 & 25.45 & 2 & 5.99 & 219 & $\mathrm{Cy}{ }^{\mathrm{a}, \mathrm{b}}$ \\
\hline 44 & SIGSTU22 & Solyc $05 g 006730.2$ & Solyc05g006730.4.1 & 1934 & 954 & 678 & 25.55 & 2 & 6.32 & 225 & $\mathrm{Cy}^{\mathrm{a}}, \mathrm{Mt}^{\mathrm{b}}$ \\
\hline 45 & SIGSTU23 & Solyc05g006740.2 & Solyc05g006740.4.1 & 1911 & 989 & 645 & 24.04 & 2 & 5.34 & 214 & $\mathrm{Cy}{ }^{\mathrm{a}}, \mathrm{Cp}^{\mathrm{a}}, \mathrm{Nu}{ }^{\mathrm{b}}$ \\
\hline 46 & SIGSTU24 & Solyc $05 g 006750.2$ & Solyc05g006750.3.1 & 1224 & 979 & 690 & 26.31 & 2 & 5.10 & 229 & $\mathrm{Cy} a, b$ \\
\hline 47 & SIGSTU25 & Solyc $05 g 026210.1$ & Solyc05g026210.2.1 & 1178 & 693 & 693 & 25.69 & 2 & 5.43 & 230 & $\mathrm{Cp}^{\mathrm{a}}, \mathrm{pm}^{\mathrm{b}}$ \\
\hline 48 & SIGSTU26 & Solyc12g097080.1 & Solyc12g097080.2.1 & 1153 & 666 & 666 & 25.85 & 2 & 6.92 & 221 & $\mathrm{Pm}^{\mathrm{a}}, \mathrm{Cy}{ }^{\mathrm{b}}$ \\
\hline 49 & SIGSTU27 & Solyc $07 g 056420.2$ & Solyc $07 g 056420.4 .1$ & 1805 & 1012 & 663 & 25.39 & 2 & 5.37 & 220 & $C y^{a, b}$ \\
\hline 50 & SIGSTU28 & Solyc $07 g 056430.2$ & Solyc07g056430.3.1 & 1811 & 1432 & 1075 & 25.71 & 2 & 5.60 & 222 & $\mathrm{Cy}{ }^{\mathrm{a}, \mathrm{b}}$ \\
\hline 51 & SlGSTU29 & Solyc $07 g 056440.2$ & Solyc $07 g 056440.3 .1$ & 1710 & 757 & 420 & 16.35 & 2 & 5.31 & 139 & $\mathrm{Cy}{ }^{\mathrm{a}, \mathrm{b}}$ \\
\hline 52 & SIGSTU30 & Solyc07g056450.2 & Solyc07g056450.3.1 & 1712 & 969 & 663 & 25.76 & 2 & 6.63 & 220 & $\mathrm{Cy}{ }^{\mathrm{a}, \mathrm{b}}$ \\
\hline 53 & SIGSTU31 & Solyc $07 g 056460.2$ & Solyc 07g056460.3.1 & 3415 & 2719 & 663 & 25.51 & 2 & 5.76 & 220 & $C y^{a, b}$ \\
\hline 54 & SIGSTU32 & Solyc $07 g 056470.2$ & Solyc07g056470.3.1 & 1024 & 951 & 663 & 25.58 & 2 & 5.57 & 220 & $\mathrm{Cy}{ }^{\mathrm{a}, \mathrm{b}}$ \\
\hline 55 & SIGSTU33 & Solyc $07 g 056480.2$ & Solyc $07 g 056480.4 .1$ & 1654 & 1054 & 660 & 25.38 & 2 & 5.42 & 219 & $\mathrm{Cy}{ }^{\mathrm{a}, \mathrm{b}}$ \\
\hline 56 & SlGSTU34 & Solyc $07 g 056490.2$ & Solyc07g056490.4.1 & 1478 & 567 & 439 & 25.54 & 3 & 7.61 & 220 & $\mathrm{Cy}{ }^{\mathrm{a}, \mathrm{b}}$ \\
\hline 57 & SlGSTU35 & Solyc07g056500.2 & Solyc $07 g 056500.3 .1$ & 1492 & 1030 & 660 & 25.41 & 2 & 6.10 & 219 & $\mathrm{Cy}{ }^{\mathrm{a}, \mathrm{b}}$ \\
\hline
\end{tabular}




\begin{tabular}{|c|c|c|c|c|c|c|c|c|c|c|c|}
\hline 58 & SIGSTU36 & Solyc07g056510.2 & Solyc 07g056510.3.1 & 1836 & 715 & 672 & 26.04 & 2 & 4.95 & 223 & $\mathrm{Cy}{ }^{\mathrm{a}, \mathrm{b}}$ \\
\hline 59 & SIGSTU37 & Solyc09g091140.2 & Solyc09g091140.4.1 & 1359 & 666 & 666 & 25.28 & 2 & 5.31 & 221 & $\mathrm{Cy}{ }^{a, b}$ \\
\hline 60 & SIGSTU38 & Solyc10g084960.2 & Solyc10g084960.2.1 & 417 & 417 & 417 & 16.37 & 1 & 6.29 & 138 & $\mathrm{Pm}^{\mathrm{a}}, \mathrm{Cy}$ \\
\hline 61 & SIGSTU39 & Solyc $08 g 062570.1$ & Solyc08g062570.1.1 & 1242 & 660 & 660 & 25.97 & 2 & 6.76 & 219 & $\mathrm{Cy} \mathrm{a}^{\mathrm{a}} \mathrm{b}$ \\
\hline 62 & SIGSTU4O & Solyc10g007620.2 & Solyc10g007620.3.1 & 1604 & 1031 & 666 & 25.84 & 2 & 5.79 & 221 & $\mathrm{Cy}{ }^{\mathrm{a}, \mathrm{b}}$ \\
\hline 63 & SIGSTU41 & Solyc01g081310.2 & Solyc01g081310.3.1 & 1531 & 1432 & 678 & 25.73 & 2 & 5.71 & 225 & $\mathrm{Cy}{ }^{\mathrm{a}, \mathrm{b}}$ \\
\hline 64 & SIGSTU42 & Solyc $06 g 069040.2$ & Solyc06g069040.4.1 & 4891 & 4499 & 681 & 25.96 & 2 & 5.15 & 226 & $\mathrm{Cy}^{\mathrm{a}}, \mathrm{Cp} \mathrm{p}^{\mathrm{b}}$ \\
\hline 65 & SIGSTU43 & Solyc $03 g 116130.1$ & Solyc03g116130.2.1 & 879 & 702 & 702 & 25.65 & 2 & 5.39 & 223 & $\mathrm{Cy}{ }^{\mathrm{a}, \mathrm{b}}$ \\
\hline 66 & SIGSTU44 & Solyc03g116120.1 & Solyc03g116120.3.1 & 740 & 675 & 675 & 25.88 & 2 & 5.93 & 224 & $\mathrm{Cy}^{\mathrm{a}, \mathrm{b}}$ \\
\hline 67 & SIGSTU45 & Solyc $02 g 081240.1$ & Solyc02g081240.1.1 & 947 & 669 & 669 & 25.52 & 2 & 5.18 & 222 & $\mathrm{Cy}^{\mathrm{a}}, \mathrm{Nu}{ }^{\mathrm{b}}$ \\
\hline 68 & SIGSTU46 & Solyc01g099590.2 & Solyc01g099590.4.1 & 1257 & 943 & 675 & 25.48 & 2 & 5.72 & 224 & $\mathrm{Cy}{ }^{\mathrm{a}, \mathrm{b}}, \mathrm{Cp}^{\mathrm{a}}$ \\
\hline 69 & SlGSTU47 & Solyc01g086680.2 & Solyc01g086680.4.1 & 1858 & 949 & 675 & 25.72 & 2 & 6.36 & 224 & $\mathrm{Cy}{ }^{\mathrm{a}, \mathrm{b}}$ \\
\hline
\end{tabular}

Notes: Cp, Chloroplast. Cy, Cytoplasm. Mt, Mitochondria. Nu, Nucleus. Pm, Plasma-membrane.

\section{Figures}

A

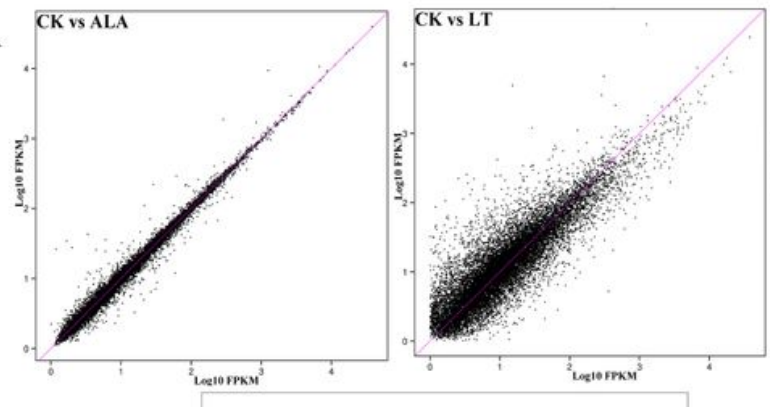

B

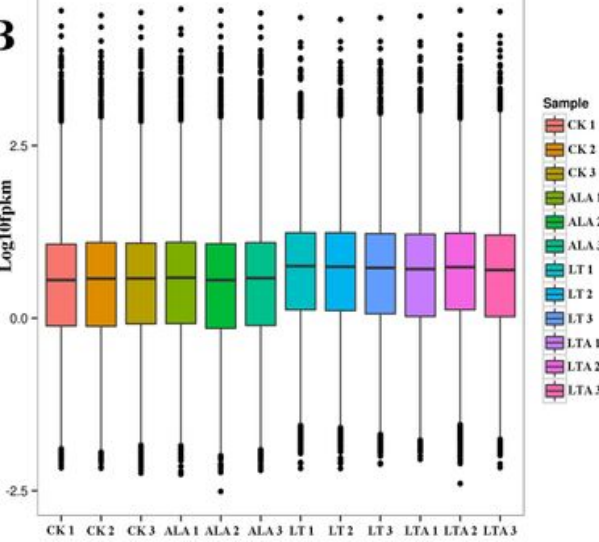

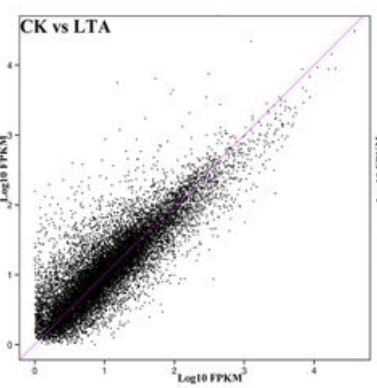
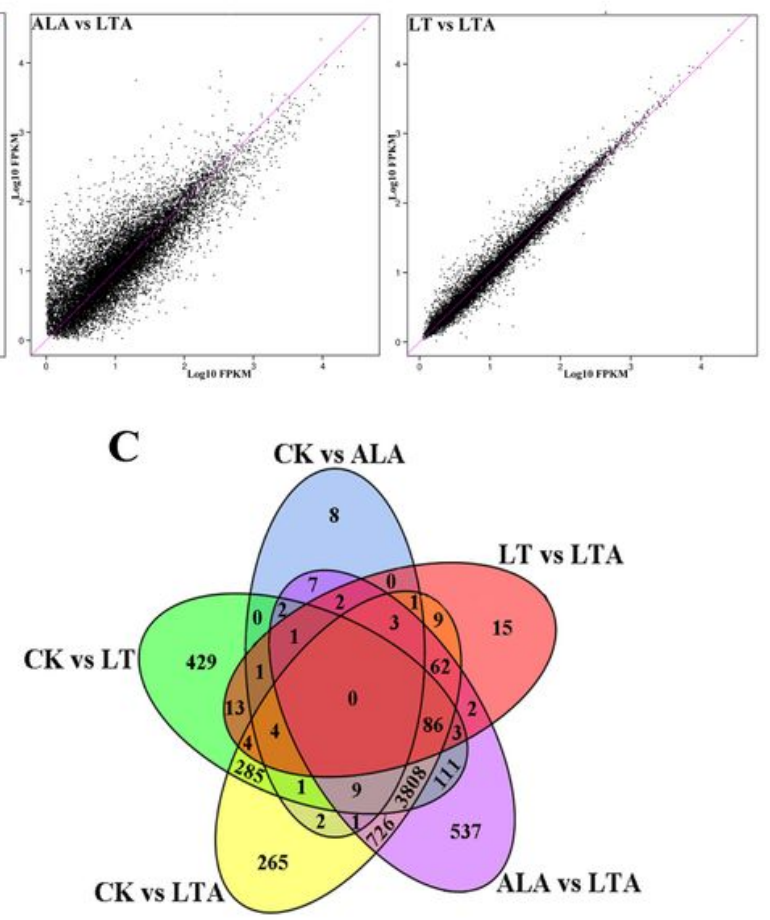

\section{Figure 1}

RNA-seq data qualification. (a) Pairwise comparison of whole gene expression levels of four sets of samples with three biological replications. (b) Boxplot of overall expression levels of four sets of samples with three biological replications. The y-axis displays the log10 (FPKM) of every sample. (c) Venn diagram showing the number of DEGs in CK vs. ALA, LT vs. LTA, ALA vs. LTA, CK vs. LTA and CK vs. LT. 

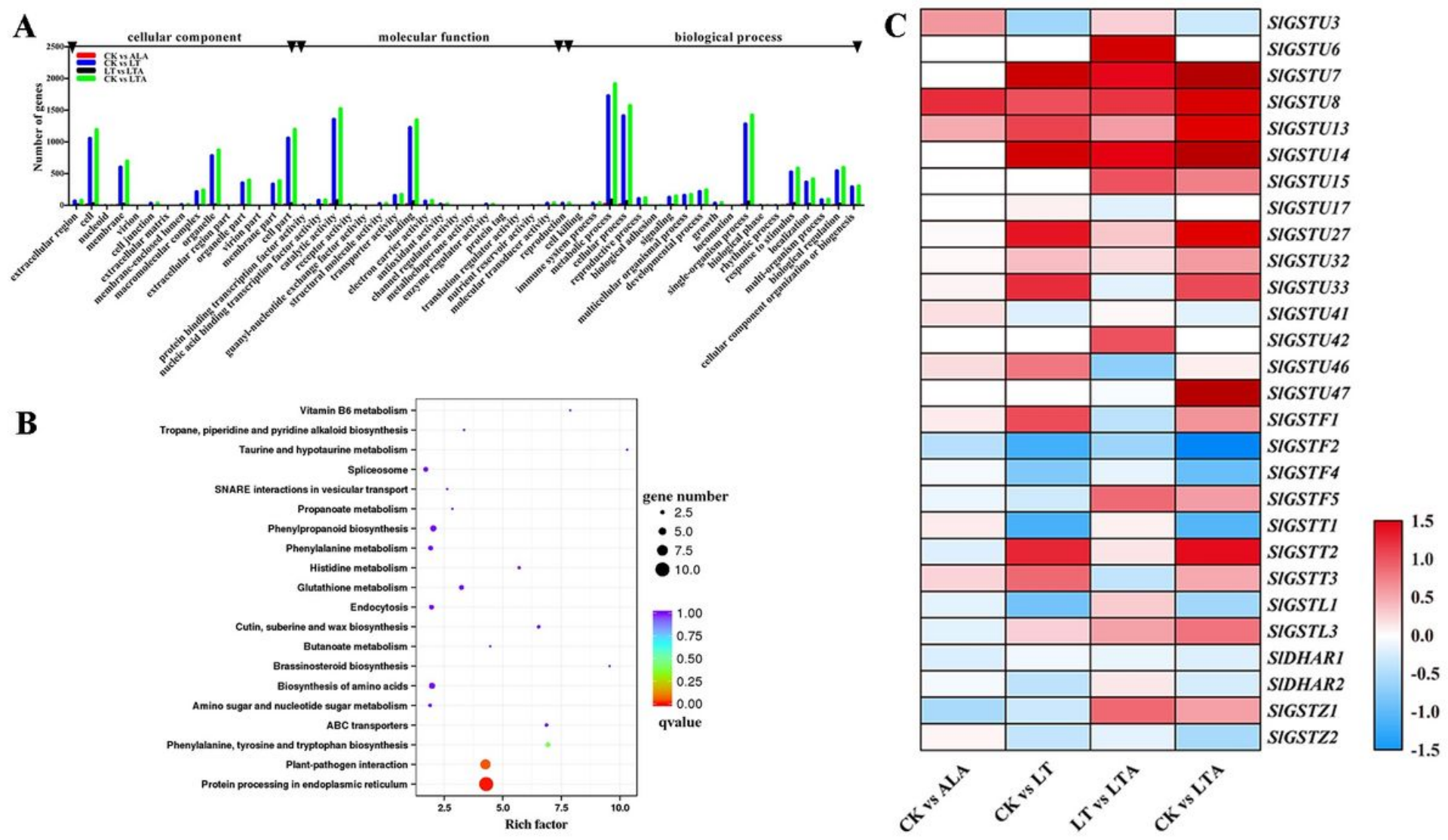

Figure 2

RNA-seq analysis of the four sets of samples. (a) GO annotation of DEGs. GO annotation of DEGs in CK vs. ALA, CK vs. LT, LT vs. LTA and CK vs. LTA. (b) KEGG pathways enrichment of DEGs. KEGG enrichment of DEGs in LT vs. LTA. (c) Expression analysis of SIGST genes in CK vs. ALA, CK vs. LT, LT vs. LTA and CK vs. LTA.

A
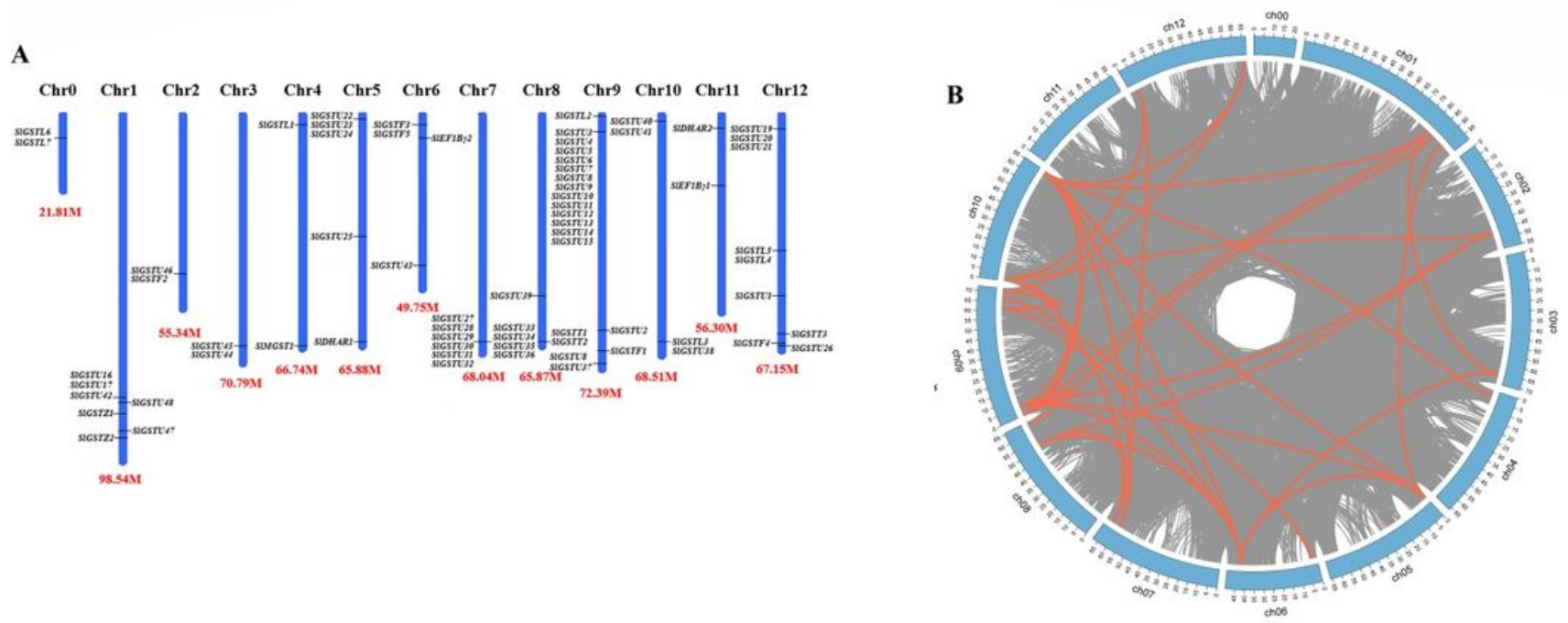


\section{Figure 3}

Chromosome mapping and tandem repeat analysis of SIGST gene. (a) Genomic distribution of SIGST genes on tomato chromosomes. The chromosome numbers and sizes $(\mathrm{Mb})$ are indicated at the top and bottom of each bar, respectively. (b) A Circos plot illustrating the physical location of SIGST genes among the tomato chromosomes. Gray ribbons represent the putative duplicated segments from the whole genome duplication. Paralog pairs of SIGST genes are linked with red lines.

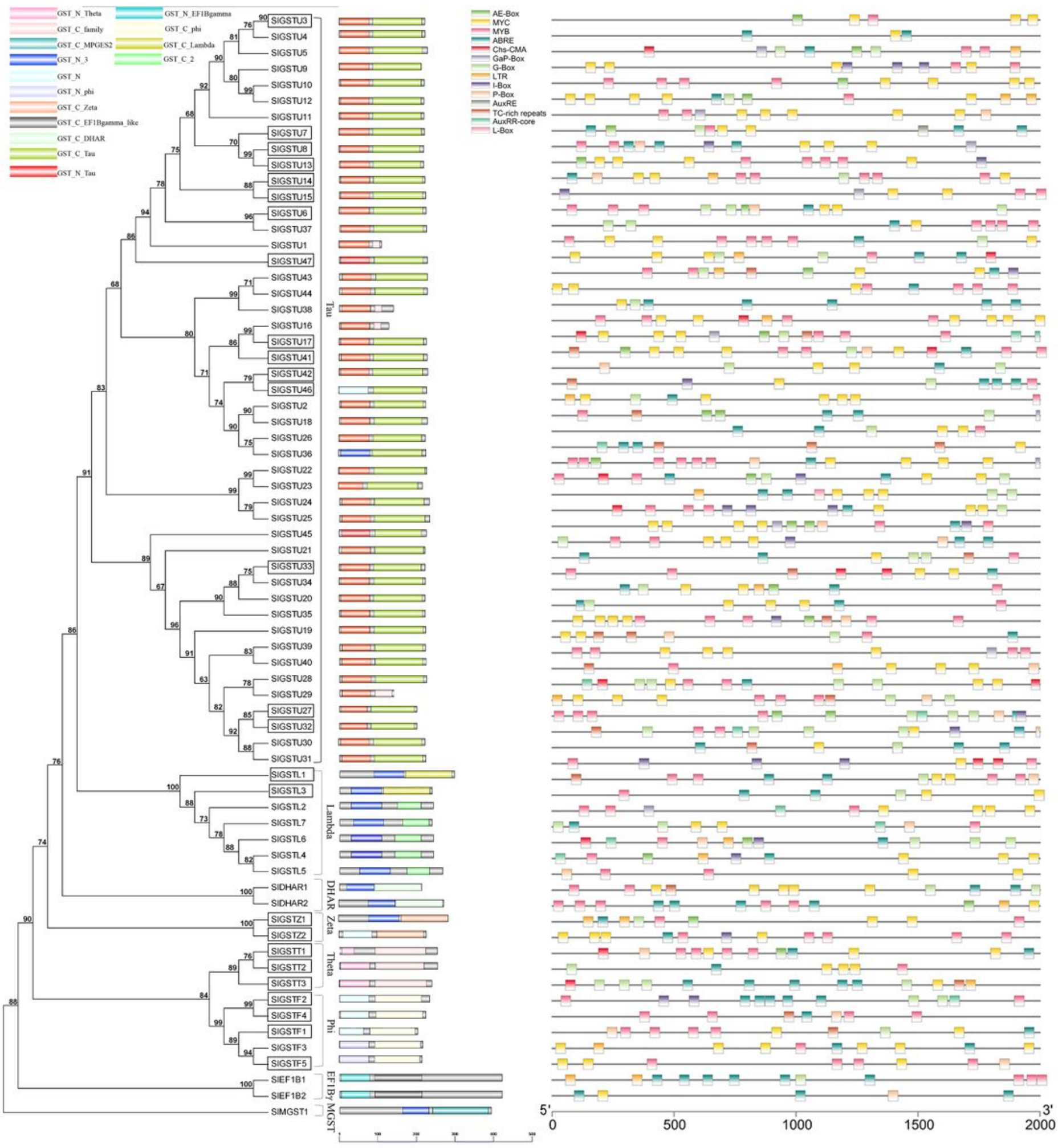




\section{Figure 4}

Analysis of protein domain and promoter. The SIGST genes circled in black box were differentially expressed in transcriptome.

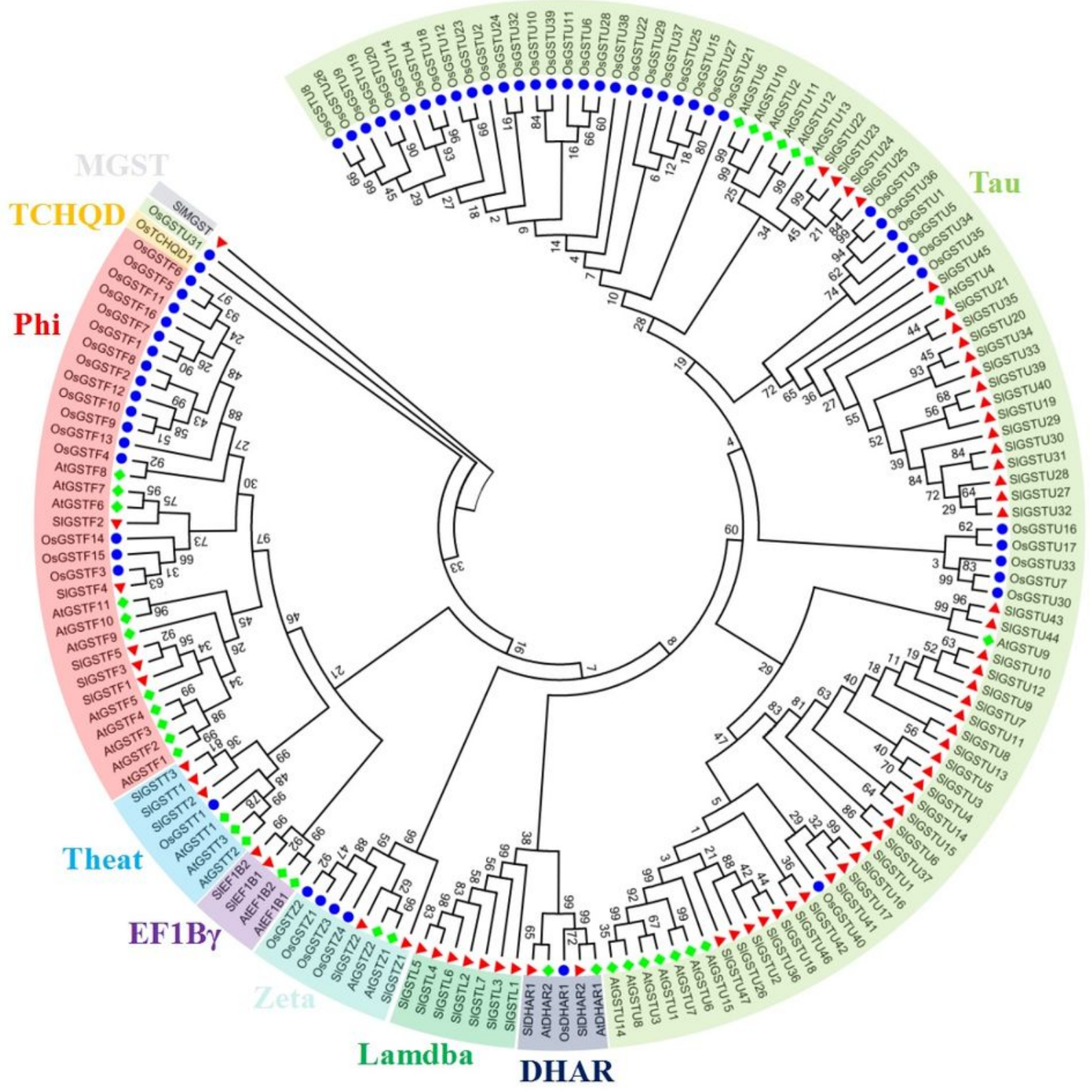

Figure 5 
Phylogenetic tree of GST genes from tomato, arabidopsis and rice. The red triangle represents the GST genes in tomato. The blue circle represents the GST genes in rice. Green diamond represents GST genes in Arabidopsis thaliana.
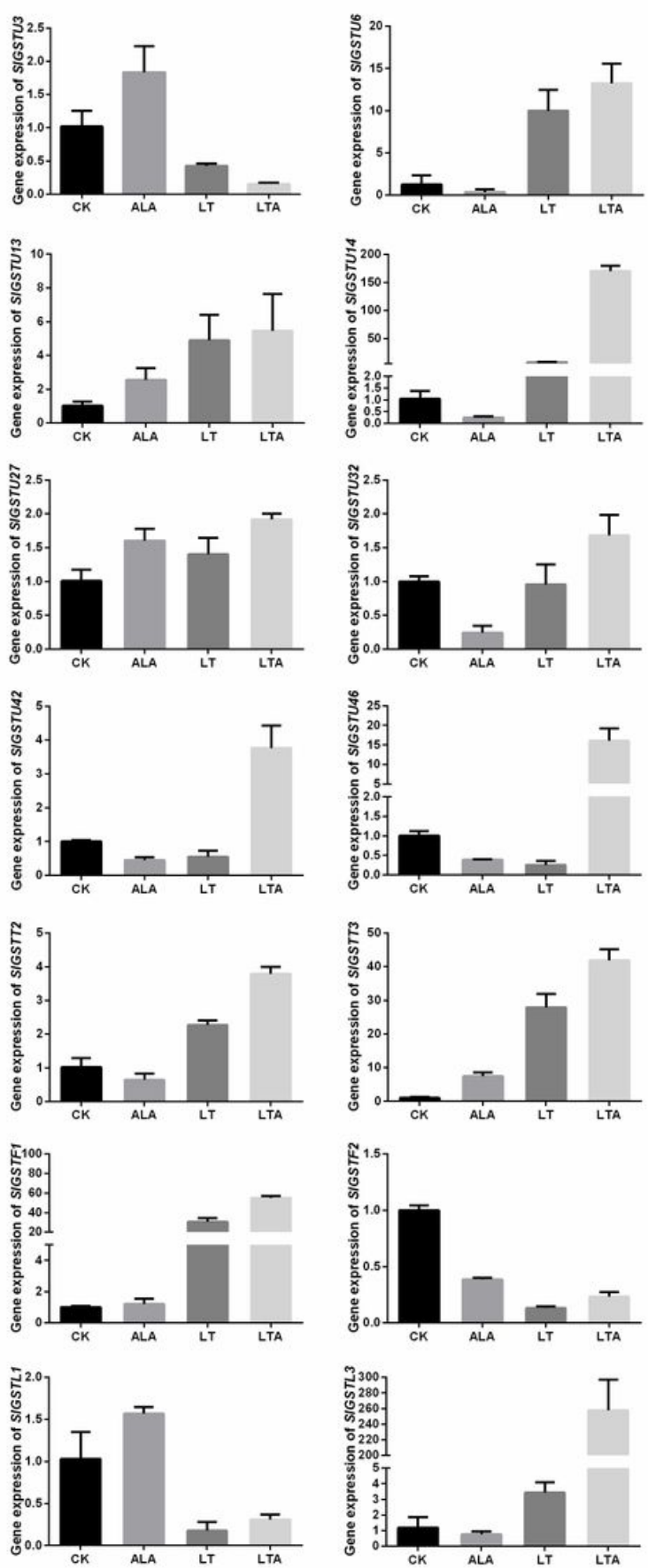
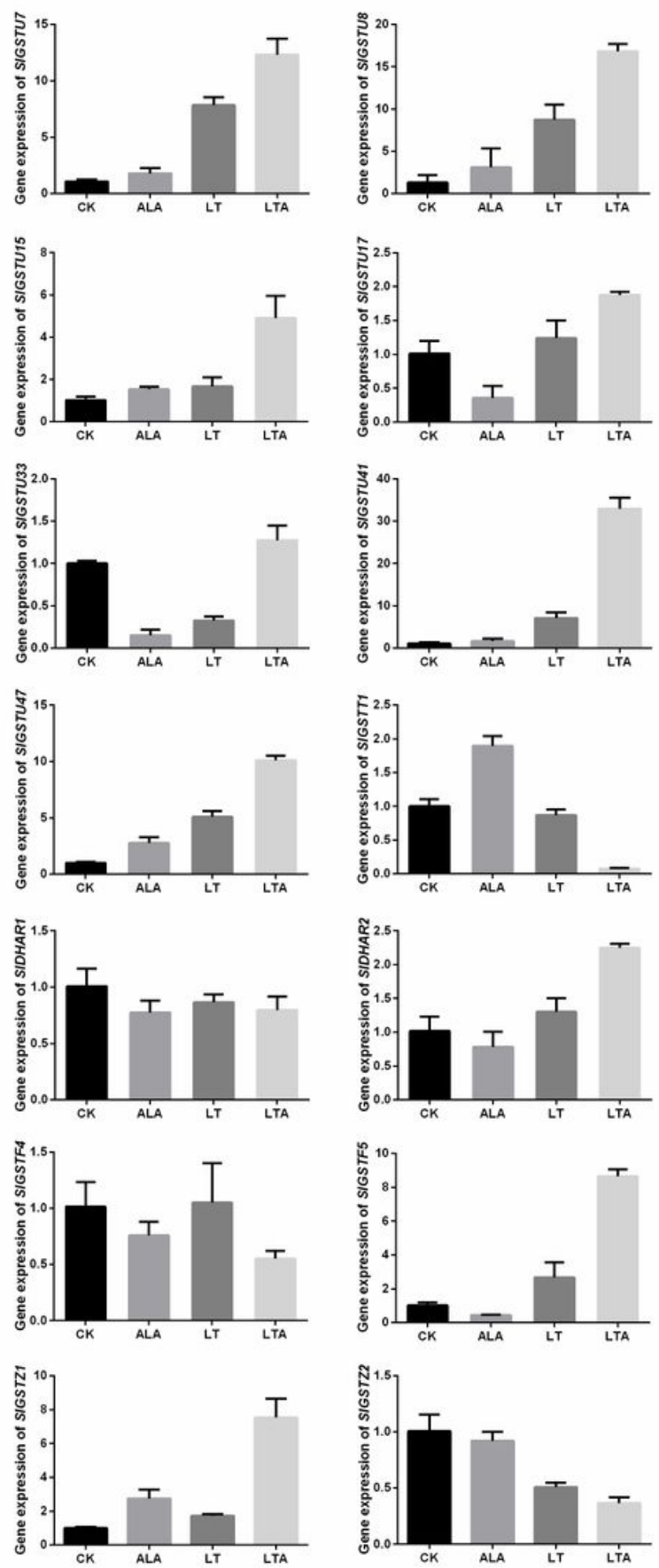

Figure 6

Expression of SIGSTs in tomato leaf under low temperature stress mediated by exogenous ALA. 


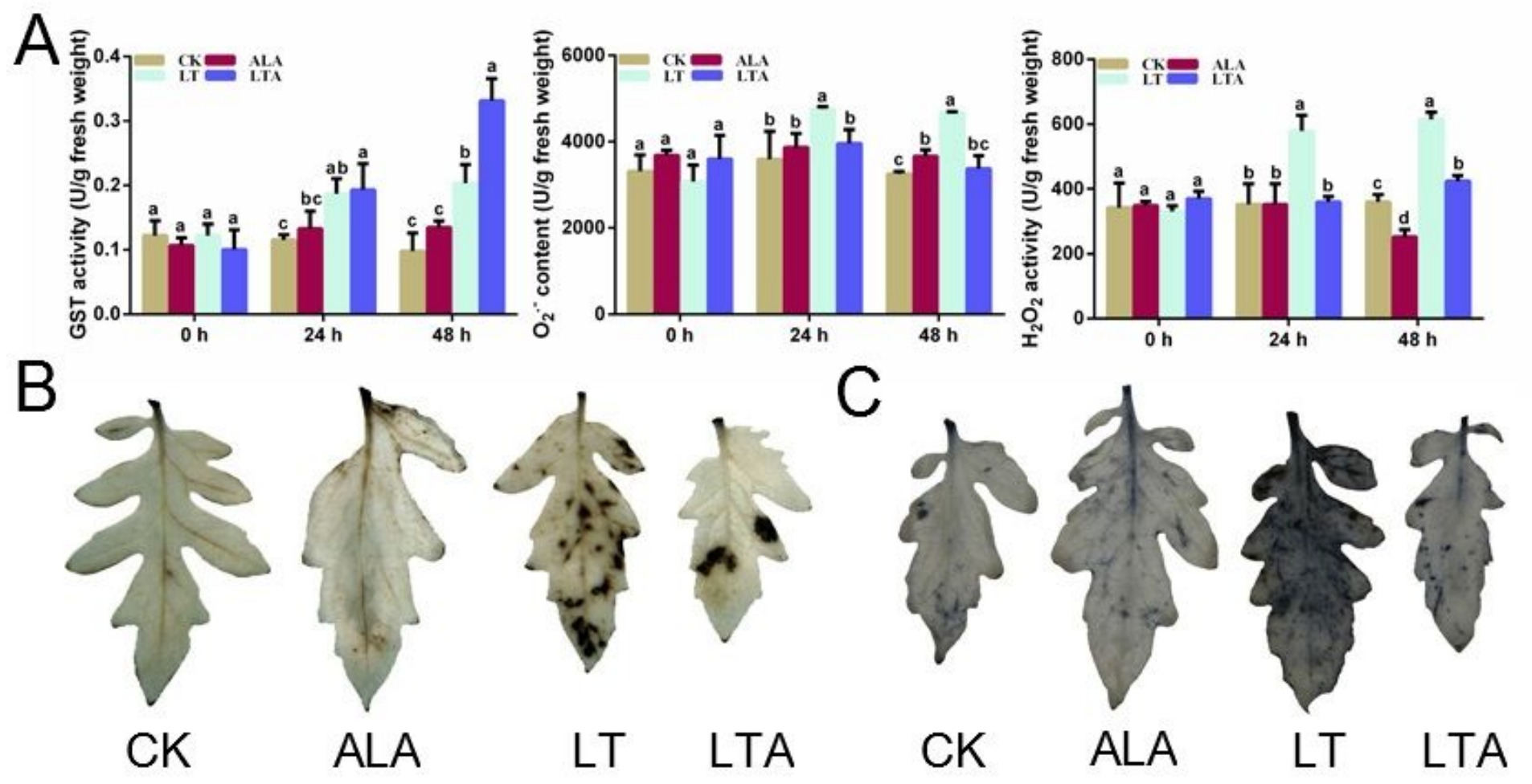

Figure 7

Determination of GST activity and analysis of ROS content. (a) Determination of physiological indicators is GST activity, $\mathrm{H} 2 \mathrm{O} 2$ content and 02.- content. The experiments were repeated for three times. Different letters above the bars indicate a significant difference determined by one-way ANOVA with Tukey's test ( $P$ < 0.05). (b) Histochemical staining of $\mathrm{H} 2 \mathrm{O} 2$ in tomato leaves at $24 \mathrm{~h}$ of treatment. The black spots represent the degree of accumulation of H2O2. (c) Histochemical staining of 02.- in tomato leaves at 24 $\mathrm{h}$ of treatment. The black spots represent the degree of accumulation of 02.-. 


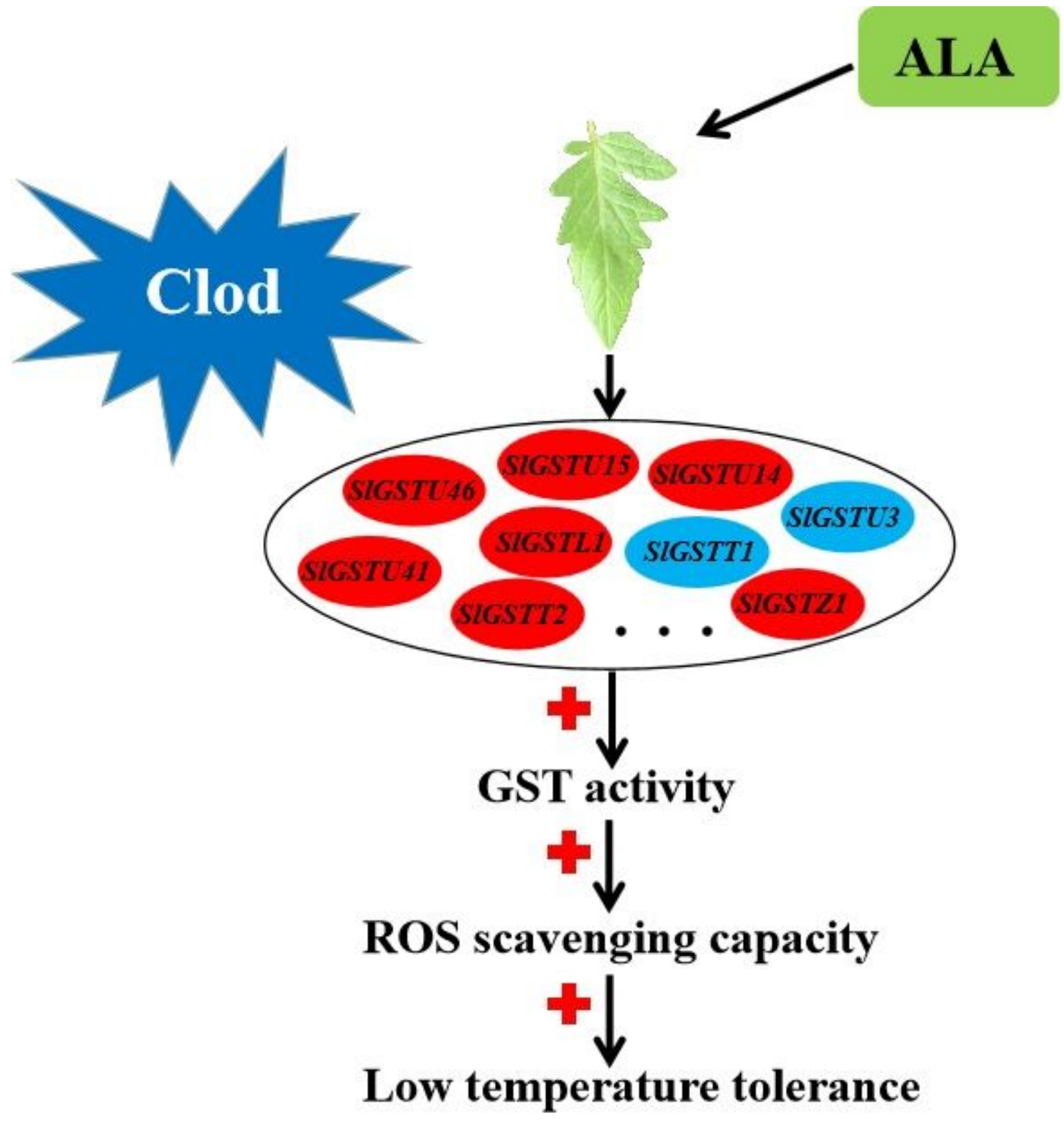

Figure 8

Exogenous application of ALA under low temperature stress increases the tomato low temperature tolerance model by inducing SIGST genes expression and GST activity. The red plus sign indicates positive regulation.

\section{Supplementary Files}

This is a list of supplementary files associated with this preprint. Click to download.

- Tables1.doc 